\title{
Functional analysis of the omega- 6 fatty acid desaturase (CaFAD2) gene family of the oil seed crop Crambe abyssinica
}

Jihua Cheng 1,2, Li-Hua Zhu³, Elma MJ Salentijn', Bangquan Huang², Jens Gruber ${ }^{4}$, Annemarie C Dechesne', Frans A Krens ${ }^{1}$, Weicong Qi ${ }^{1,2}$, Richard GF Visser ${ }^{1}$ and Eibertus N van Loo ${ }^{1 *}$

\begin{abstract}
Background: Crambe abyssinica produces high erucic acid (C22:1, 55-60\%) in the seed oil, which can be further increased by reduction of polyunsaturated fatty acid (PUFA) levels. The omega- 6 fatty acid desaturase enzyme (FAD2) is known to be involved in PUFA biosynthesis. In crambe, three CaFAD2 genes, CaFAD2-C1, CaFAD2-C2 and CaFAD2-C3 are expressed.

Results: The individual effect of each CaFAD2 gene on oil composition was investigated through studying transgenic lines (CaFAD2-RNAi) for differential expression levels in relation to the composition of seed-oil. Six first generation transgenic plants $\left(T_{1}\right)$ showed C18:1 increase (by $6 \%$ to 10.5\%) and PUFA reduction (by 8.6\% to 10.2\%). The silencing effect in these $T_{1}$-plants ranged from the moderate silencing (40\% to $50 \%$ reduction) of all three CaFAD2 genes to strong silencing (95\% reduction) of CaFAD2-C3 alone. The progeny of two T1-plants (WG4-4 and WG19-6) was further analysed. Four or five transgene insertions are characterized in the progeny $\left(T_{2}\right)$ of WG19-6 in contrast to a single insertion in the $T_{2}$ progeny of WG4-4. For the individual $T_{2}$-plants of both families (WG19-6 and WG4-4), seed-specific silencing of CaFAD2-C1 and CaFAD2-C2 was observed in several individual $T_{2}$-plants but, on average in both families, the level of silencing of these genes was not significant. A significant reduction in expression level $(P<0.01)$ in both families was only observed for CaFAD2-C3 together with significantly different C18:1 and PUFA levels in oil.
\end{abstract}

Conclusions: CaFAD2-C3 expression is highly correlated to levels of C18:1 $(r=-0.78)$ and PUFA $(r=0.75)$, which suggests that CaFAD2-C3 is the most important one for changing the oil composition of crambe.

Keywords: Crambe abyssinica, Fatty acid desaturase 2, Oil crop, Oleic acid, Polyunsaturated fatty acid, RNAi, Gene expression

\section{Background}

Crambe (Crambe abyssinica Hochst ex. R. E. Fr.) belongs to the Brassicaceae plant family. The seed oil of crambe contains a high content of erucic acid (C22:1, $55 \%$ to $60 \%$ ) and this oil thus has applications as industrial oil [1]. Erucic acid in form of erucamide, a major derivative from C22:1, can be used as slip agent in plastics, or lubricants, nylon and cosmetics [2]. More recently, crambe oil is considered to be potential to produce biofuel

\footnotetext{
* Correspondence: robert.vanloo@wur.nl

${ }^{1}$ Wageningen UR Plant Breeding, P.O. Box 16,6700, AA Wageningen, The Netherlands

Full list of author information is available at the end of the article
}

[3]. The cultivation of crambe yields up to $1 \mathrm{t} \mathrm{ha}^{-1}$ of oil, comparative to that of high erucic acid rapeseed (HEAR). Furthermore, the processing costs for crambe oil extraction are in the same range of that for rapeseed $[1,4,5]$. The high yield and the fact that crambe is not able to cross with food oil crops in nature make crambe an ideal platform to produce industrial oils [6,7]. In addition to high erucic acid (C22:1), crambe oil contains also certain amounts of oleic acid (C18:1), linoleic acid (C18:2) and linolenic acid (C18:3).

The value of crambe oil can be further improved not only by increasing the C22:1 content but also by reducing the content of polyunsaturated fatty acids (PUFA,

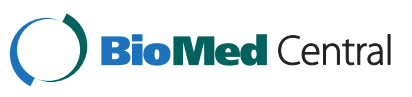


$\mathrm{C} 18: 2+\mathrm{C} 18: 3)$. Firstly, an increase in C22:1 can reduce the purification cost of $\mathrm{C} 22: 1$ from $\mathrm{C} 18$-fatty acids. It was estimated that $10 \%$ increase of $\mathrm{C} 22: 1$ in oil would reduce the processing costs by half [8]. Secondly, reduction in PUFA is beneficial for storage and extending the shelf life of the oil because PUFA are highly prone to oxidation during storage[9]. Thirdly, high PUFA content causes higher viscosity for the oil (a disadvantage to biodiesel) [10]. Considerable efforts have been made to reduce PUFA in oil. A common chemical approach is to reduce the PUFA content or increase the C18:1 content through hydrogenation $[9,11]$. However, this hydrogenating process is expensive and adds extra 2 to 3 cent per pound cost to the price of oil [12].

Molecular breeding approaches to change composition of seed oil are targeting important genes involved in the fatty acid biosynthesis pathways, which have been intensively studied and many genes involved have been characterized. In short, $\mathrm{C} 18: 1$ is de novo synthesized in plastids and transported into the endoplasmic reticulum where the C18:1 is incorporated into phosphatidylcholine $(\mathrm{PC})$ and may undergo desaturation to $\mathrm{C} 18: 2$ and next to $\mathrm{C} 18: 3$ by the actions of two microsomal enzymes; delta-12-fatty acid desaturase (FAD2) [omega-6 desaturase] and delta-15-fatty acid desaturase (FAD3) [omega-3 desaturase], respectively [9,13]. Alternatively, C18:1 may undergo elongation to very long chain fatty acids (VLCFA, C22:1 for example) by the action of fatty acid elongation (FAE) complex [2,14-17]. Mutant and genetic mapping studies showed that the enzyme FAD2 was found to be mainly responsible for C18:1 and PUFA content although FAD3 also contributes to a limited degree to these traits $[9,18]$. In addition, the acyl flux between the two pathways, the prokaryotic (plastidial) and eukaryotic (mainly in the ER) pathway, is influencing the C18:1 pool [19-21].

Genetic modification (GM) aimed at regulating the FAD2 expression has been applied to produce oils with higher C18:1 in various oil crops [8,22,23]. For example, by anti-sense suppression of FAD2 in Brassica juncea, a transgenic line was obtained that produced oil with higher C18:1 (73\%) and lower PUFA (8\% of C18:2; 9\% of C18:3) compared to the wild type (53\% of C18:1; $24 \%$ of C18:2; $16 \%$ of C18:3) [23]. Similarly, a significant change in C18:1 and PUFA and even a C22:1 increase in the seed oil was observed when silencing FAD2 with both co-suppression and anti-sense in Brassica carinata [8]. Gene silencing by RNAi has been considered to be a particularly efficient way to obtain stable transgenic plants with the silenced target genes [24,25]. RNAi-mediated silencing of GhFAD2 in cotton enabled over $60 \%$ increase in C18:1 [22]. Simultaneous RNAi-mediated silencing of FAD2 and FAE1 in Brassica napus caused not only significant increase in C18:1 (from $62 \%$ to $85 \%$ ), but also reduction in C22:1 and PUFA (from 26\% to $10 \%$ and from $0.87 \%$ to $0 \%$ respectively) [26]. Recently, several efficient protocols for crambe transformation are available $[27,28]$ and RNAi has shown to be an effective gene knockdown tool for crambe where CaFAD2 RNAi gene silencing resulted in increased C18:1 levels (from $14.5 \%$ to $24.9 \%$ ) [29]. Introduction of two heterologous genes, LdLPAAT and $B n F A E 1$, in such CaFAD2-RNAi lines directed the oil biosynthesis towards the incorporation of $\mathrm{C} 22: 1$ at the $s n-2$ position of triacylglycerol, thereby the C22:1 level increased from $60 \%$ in the wild type to $73 \%$ in the best transgenic crambe line [29].

An obstacle to adaptation of such genetic modified (GM) crops is the lack of broad acceptance by a part of the community in many countries [30]. Furthermore, there are some cases where RNAi-mediated traits are not completely reliable on the long term in generating stable target gene suppression [25]. Currently, breeding of the allo-hexaploid crambe mainly relies on traditional approaches, however, the possibilities are restricted by the lack of genetic variation for important agronomic traits $[1,31,32]$. In such situations and particularly when genes controlling a phenotype are known, mutation breeding of induced or natural mutations, identified via “TILLING" (Targeting Induced Local Lesions IN Genomes) [33], offers a reliable, stable, non-GM approach to obtain the desired oil quality in crambe. A drawback is that "TILLING" is still a challenging task in polyploid crops, where the multiple alleles are creating problems in identifying desirable genetic changes due to gene redundancy. Despite these problems successful cases of targeted mutagenesis have been reported in polyploid crops [34-38]. For instance, targeted mutation breeding of natural or induced variation in the FAD2 gene or mining natural variants has been used to develop crops with high C22:1 or less PUFA [38-40]. By combining mutations or natural variants of $F A D 2$ and $F A D 3$, it was possible to produce oils with higher C18:1 and lower C18:3 in soybean and Brassica napus $[9,11]$. In the allo-hexaploid genome of Crambe abyssinica cv. 'Galactica' seven FAD2 genes are present, of which only three are transcriptionally active throughout plant development (CaFAD2-C1, CaFAD2-C2 and CaFAD2-C3) [32]. The possibility of functional redundancy among the active $C a F A D 2$ family members may complicate the gain of crambe lines with desirable oil composition via mutation breeding.

The aim of the present study is to specify which of the $F A D 2$ genes in crambe is the key gene for increasing the C18:1 level, but reducing the PUFA content. FAD2-RNAi lines of crambe cv. 'Galactica' were studied for functional correlations between the individual CaFAD2 family members, CaFAD2-C1, CaFAD2-C2 and CaFAD2-C3 and seed-oil composition. Seed-oil composition and gene expression studies were performed in two independent 
families of the second generation transgenic lines $\left(\mathrm{T}_{2^{-}}\right.$ plants). In addition, two other genes, CaFAD3 and CaFAE1 that are acting in close connection to $C a F A D 2$, were also involved in the study.

\section{Results \\ CaFAD2-RNAi lines}

We have previously shown that the major expressed CaFAD2 gene in developing crambe seeds is CaFAD2-C3, while the other two genes CaFAD2-C1 and CaFAD2-C2 are naturally expressed to much lower levels (4 and 100 times lower than CaFAD2-C3 respectively) [32]. A DNA fragment of $C a F A D 2-C 2$, sharing $97 \%$ and $96 \%$ nucleotide identity with CaFAD2-C1 and CaFAD2-C3 respectively, was used to trigger RNAi-mediated silencing of the CaFAD2 genes in crambe. Two primary transgenic plants $\left(\mathrm{T}_{0}\right.$ generation,WG4 and WG19) that showed significantly lower contents of $\mathrm{C} 18: 2$ and $\mathrm{C} 18: 3$, but higher level of $\mathrm{C} 18: 1$ in the seed-oil were used in this study [29]. For these two independent transgenic lines, the effect of RNAi-silencing on the expression of the individual CaFAD2 genes and the composition of the seed-oil were analysed for the seeds produced by the first generation $\left(T_{1}\right)$ and the second generation $\left(T_{2}\right)$. In the $T_{2}$ generation the transgene copy number was determined and the relative gene-expression of the individual genes was correlated to differences in seed-oil composition to determine the effect of silencing of the individual CaFAD2 gene(s).

\section{CaFAD2 gene-silencing and oil composition in $\mathrm{T}_{1}$-generation}

Among six individual plants analysed, simultaneous but moderate silencing of all three CaFAD2 genes $(40 \%$ to $50 \%$ reduction) was found only in one plant, WG4-5. In two plants WG19-5 and WG19-6 no signs of genesilencing was observed. In the remaining three plants (WG4-3, WG4-4 and WG19-4) no silencing was detected for CaFAD2-C1 and CaFAD2-C2 whereas CaFAD2-C3 was silenced to different levels whereby a strong silencing of CaFAD2-C3 (95\% reduction) was detected in plant WG4-4 followed by a moderate level of CaFAD2-C3 silencing (40\% to 50\% reduction) in WG4-3 and WG19-4 (Figure 1). To measure the final effect of gene-silencing, the oil composition was determined for around 20 single ripe seeds per plant. Despite the fact that clear silencing was only detected in the developing seeds of one plant (WG4-4), the contents of C18:1, C18:2 and C18:3 in the seeds of all six plants were significantly different to that of the control (Table 1). The C18:1 content was found to be $6 \%$ to $10.5 \%$ above the control, and the content of PUFA $(\mathrm{C} 18: 2+\mathrm{C} 18: 3)$ was a concomitant $4.3 \%$ to $9.2 \%$ lower than the control. Regarding C22:1 content, the plants WG4-3, WG4-4 and WG19-6 scored significantly higher than the control by respectively $2.4 \%(P<0.01), 3.9 \%(P<0.01)$ and $3.1 \%(P<0.01)$ whereas the remaining three plants showed no significant difference to the control (Table 1).

An explanation for the fact that the level of CaFAD2 silencing and the oil composition are not clearly correlated

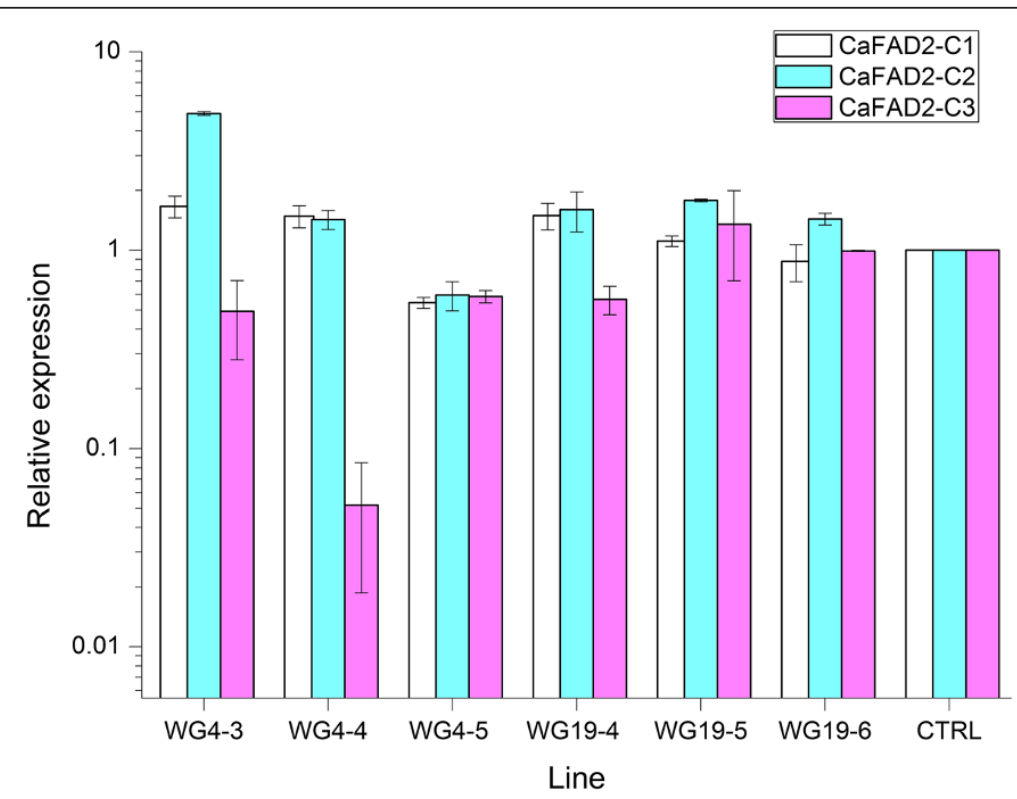

Figure 1 Relative expression levels of CaFAD2 gene family members in the seeds of $T_{1}$ plants of $C$. abyssinica. Six $T_{1}$ plants (WG4-3, WG4-4, WG4-5, WG19-4, WG19-5 and WG19-6) and a control (CTRL) were measured. Qantitification was performed by qPCR for bulk seeds (five to ten, 20 DAP) of each plant. The relative expression levels in the seeds of $T_{1}$ plants were calculated with $2 \wedge-\triangle \triangle C t$ method using $\beta$-ACT2 as a reference gene. 
Table 1 Oil composition (\%) in seeds of $T_{1}$ lines of $C$. abyssinica

\begin{tabular}{|c|c|c|c|c|c|c|}
\hline ID & No. of seed & FA level & Oleic acid (C18:1) & Linoleic acid (C18:2) & Linolenic acid (C18:3) & Erucic acid (C22:1) \\
\hline \multirow[t]{3}{*}{ WG4-3 } & 18 & Mean $\pm \mathrm{SD}^{\mathrm{a}}$ & $20.1 \pm 2.4^{* *}$ & $2.7 \pm 0.9^{* *}$ & $3.0 \pm 0.8^{* *}$ & $65.5 \pm 3.4^{* *}$ \\
\hline & & Max & 26.3 & 4.7 & 5.4 & 69.3 \\
\hline & & Min & 16.8 & 1.6 & 1.9 & 54.6 \\
\hline \multirow[t]{3}{*}{ WG4-4 } & 20 & Mean \pm SD & $20.0 \pm 1.1^{* *}$ & $2.3 \pm 0.5^{* *}$ & $3.0 \pm 0.5^{* *}$ & $67 \pm 2.5^{* *}$ \\
\hline & & Max & 21.9 & 3.3 & 4.1 & 70.8 \\
\hline & & Min & 18.0 & 1.5 & 2.3 & 60.7 \\
\hline \multirow[t]{3}{*}{ WG4-5 } & 19 & Mean \pm SD & $21.9 \pm 3.5^{* *}$ & $2.1 \pm 1.8^{* *}$ & $2.3 \pm 0.9^{* *}$ & $63.5 \pm 3.2$ \\
\hline & & Max & 27.4 & 7.6 & 5.4 & 66.6 \\
\hline & & Min & 11.5 & 1.0 & 1.5 & 55.5 \\
\hline \multirow[t]{3}{*}{ WG19-4 } & 19 & Mean \pm SD & $22.6 \pm 2.3^{* *}$ & $1.6 \pm 0.2^{* *}$ & $2.5 \pm 0.3^{* *}$ & $60.7 \pm 5.2$ \\
\hline & & Max & 29.5 & 2.1 & 3.1 & 65.9 \\
\hline & & Min & 20.4 & 1.3 & 1.8 & 46.1 \\
\hline \multirow[t]{3}{*}{ WG19-5 } & 20 & Mean $\pm S D$ & $21.4 \pm 3.2^{* *}$ & $2.7 \pm 1.8^{* *}$ & $3.3 \pm 0.7^{*}$ & $60 \pm 4.6$ \\
\hline & & Max & 27.1 & 9.5 & 5.3 & 64.1 \\
\hline & & Min & 11.3 & 1.3 & 2.3 & 50.2 \\
\hline \multirow[t]{3}{*}{ WG19-6 } & 20 & Mean \pm SD & $18.0 \pm 0.9^{*}$ & $2.0 \pm 0.4^{* *}$ & $2.4 \pm 0.3^{* *}$ & $66.2 \pm 1.0^{* *}$ \\
\hline & & Max & 20.0 & 2.9 & 3.0 & 68.0 \\
\hline & & Min & 16.4 & 1.4 & 1.9 & 64.5 \\
\hline \multirow[t]{3}{*}{ Control } & 19 & Mean \pm SD & $12.0 \pm 0.5$ & $8.2 \pm 0.4$ & $5.1 \pm 0.2$ & $63.1 \pm 0.8$ \\
\hline & & Max & 12.8 & 9.0 & 5.6 & 64.4 \\
\hline & & Min & 10.9 & 7.5 & 4.7 & 61.6 \\
\hline
\end{tabular}

The fatty acid composition in single seed was measured, and for each line several seeds were used for measurement. Kruskal-Wallis test was run to determine significance level of difference between the FAD2-RNAi lines and the control. a: $S D=$ standard deviation. Significance levels: ${ }^{*} P<0.05$ and ${ }^{* *} P<0.01$.

in $\mathrm{T}_{1}$ generation may reside in differences in zygosity level of the transgene copies among the $\mathrm{T}_{1}$-plants. As the expression profile was analysed on bulks of five to ten seeds, variation in transgene copy number and the presence of seeds that have segregated to the wild-type may mask the silencing effect. Indeed, among the fatty acid profiles of the individual seeds of WG4-5 and WG19-5 segregation to the wild-type oil composition was observed (Additional file 1).

\section{CaFAD2 silencing and oil composition in $\mathrm{T}_{2}$-generation}

Two $\mathrm{T}_{1}$-plants (WG4-4 and WG19-6) with stable and high C18:1 content were chosen to develop a second generation for an extensive study of the inheritance of the "high C18:1, low PUFA" oil-phenotype and to examine the correlation between silencing of the respective CaFAD2 genes, the oil composition and the expression of two other genes involved in the seed oil biosynthesis (CaFAD3 and CaFAE1).

\section{Transgene copy-number}

Seventeen $\mathrm{T}_{2}$-plants (5 from WG4-4 and 12 from WG19-6, designated as family WG4-4 and WG19-6) were randomly selected and characterised for the number of transgene insertion by Southern analysis. Four or five transgene insertions were detected in the progeny of WG19-6 with a similar pattern. In contrast, a single insertion was detected in all five analysed progeny plants of WG4-4 (Additional file 2).

\section{CaFAD2 gene-silencing}

To test whether and to what extent RNAi-mediated gene-silencing was effective in the $\mathrm{T}_{2}$-plants, the expression levels of CaFAD2-C1, CaFAD2-C2 and CaFAD2-C3 in developing seeds (five to ten bulked seeds per plant, 20 DAP) were quantified for all twenty-two $T_{2}$-plants by qRT-PCR. The relative expression levels were calculated relative to the average expression level of the respective genes in the control. Collectively, down-regulation of gene expression is clearly detectable in the $\mathrm{T}_{2}$-plants of both $\mathrm{T}_{2}$ families, WG4-4 and WG19-6, differential patterns of silencing are observed for all three CaFAD2 genes (Figure 2A). The major CaFAD2 gene expressed in crambe seed, CaFAD2-C3, is strongly silenced (> 50\% reduction) in all seven $\mathrm{T}_{2}$-plants of the WG4-4 family and in 14 out of $15 \mathrm{~T}_{2}$-plants of the WG19-6 family. Only in a single plant, WG19-6-10, minor silencing of CaFAD2-C3 is observed ( $10 \%$ reduction) (Figure $2 \mathrm{~A})$. 
In contrast to the constant and strong silencing of CaFAD2-C3, more variation in silencing is found for CaFAD2-C2 and CaFAD2-C1 in both families. In the single copy transgenic $\mathrm{T}_{2}$-plants of the WG4-4 family, CaFAD2-C2 showed strong silencing in almost half (3 out of 7) of the WG4-4 family (WG4-4-1, WG4-4-2 and WG4-4-4) whereas in the other $\mathrm{T}_{2}$-plants of WG4-4 this gene was not silenced (Figure 2A). In the other family, WG19-6, $60 \%$ of the $\mathrm{T}_{2}$-plants ( 9 out of 15 ) showed strong silencing of CaFAD2-C2 whereas the rest showed zeroto moderate silencing of CaFAD2-C2. Also, CaFAD2-C1 showed various silencing levels in both $\mathrm{T}_{2}$ families with strong silencing in three WG4-4 $\mathrm{T}_{2}$-plants (3 out of 7; $43 \%$ ) and five WG19-6 $\mathrm{T}_{2}$-plants (5 out of $15 ; 33 \%$ ) (Figure 2A).

The average expression levels of the respective genes were calculated for each $\mathrm{T}_{2}$ family in comparison with the control. In both families (WG4-4 and WG19-6), only the expression of CaFAD2-C3 is constantly and significantly different to the average control level (Figure $2 \mathrm{~B}$ ). For CaFAD2-C1 and CaFAD2-C2, the average expression levels in both families are not significantly different from the average expression level observed in the control (Figure 2B).

\section{Inheritance of CaFAD2 silencing}

As compared over two generations $\left(\mathrm{T}_{1}\right.$ and $\left.\mathrm{T}_{2}\right)$, the level of silencing in WG19-6, the line which carries multiple transgene insertions, increased from zero silencing in the $\mathrm{T}_{1}$ to $\sim 40 \%, 60 \%$ and $70 \%$ reduction for respectively CaFAD2-C1, $-C 2$ and $-C 3$ in the $\mathrm{T}_{2}$. Over two generations, $\mathrm{T}_{1}$ and $\mathrm{T}_{2}$, of the single copy transgene WG4-4 the expression patterns of the CaFAD2 genes were similar. In both generations, only CaFAD2-C3 was dominantly down-regulated in expression (Figure 1 and $2 \mathrm{~B}$ ).

\section{Effect of CaFAD2 silencing on CaFAD3 and CaFAE1 expression}

As a consequence of CaFAD2 gene silencing, and accompanying changes in the substrate flow in the oil biosynthesis pathway, the expression of other genes acting in the pathway may change. Therefore, the expression of two genes, $F A D 3$, involved in the conversion of C18:2 to $\mathrm{C} 18: 3$, and $F A E 1$, acting in the production of very long chain fatty acids (i.e. C22:1) by chain-elongation of $\mathrm{C} 18: 1$, were studied in the two $\mathrm{T}_{2}$ families (WG4-4 and WG19-6) and the control. Both genes showed differential expression among the individual plants within families, but on average no significant differences were observed between 
the two $\mathrm{T}_{2}$ families and the control (Figure 2B). Notably, CaFAD3 and CaFAE1 showed a similar regulation pattern because both of them were up- or down-regulated within the same plant with exception of two plants, WG19-6-11 and WG19-6-12 (Figure 2A).

\section{Oil composition}

The observed changes in expression pattern of the target genes resulted in significant changes in seed oil composition in $21 \mathrm{~T}_{2}$-plants studied (7 plants derived from WG44 and 14 from WG19-6). In accordance with the results found in the $\mathrm{T}_{1}$ generation, the contents of C18:1, C18:2 and $\mathrm{C} 18: 3$ showed significant differences to the control $(P<0.01)$. However, the high $C 22: 1$ content found in the oil of two $\mathrm{T}_{1}$-plants (WG4-4 and WG19-6) was not observed throughout the entire $\mathrm{T}_{2}$ offspring of these plants. The C22:1 content showed significant differences to the control in only four $\mathrm{T}_{2}$-plants (WG19-6-3, WG19-6-5, WG19-6-7, WG19-6-8) in which C22:1 levels were about $2 \%$ higher $(P<0.05)$ than the control level $(C 22: 1 \%=$ $62.5 \%)$. Compared to the control $(\mathrm{C} 18: 1 \%=12.9 \%)$, the
C18:1 contents in the oil of all the $\mathrm{T}_{2}$-plants were clearly higher $(P<0.01)$ and ranged from $24.3 \%$ to $18.2 \%$, and most of the $\mathrm{T}_{2}$-plants $(81 \%, 17$ out of 21$)$ showed a C18:1 content of more than 20\% (Figure 3A and C). Consequently, the PUFA $(\mathrm{C} 18: 2+\mathrm{C} 18: 3)$ contents in these plants were lower $(P<0.01)$ than the control $($ PUFA\% $=$ $13.8 \%)$ and ranged from $3.4 \%$ to $7.9 \%$, and in $67 \%(14$ out of 21) of the plants the PUFA content was below 5\% (Figure 3B and D).

Overall, seeds from the two $\mathrm{T}_{2}$ families (WG4-4 and WG19-6) contained different C18:1 and PUFA contents compared to the control (higher or lower respectively) (Figure 4). Among both $\mathrm{T}_{2}$ families, the content of C18:1, C18:2, C18:3, C22:1 and PUFA showed no significant difference (Figure 4). However, family WG19-6 showed more variation in both C18:1 and PUFA contents than family WG4-4 (Additional file 3).

\section{Correlation between gene expression and oil composition}

To investigate the relationship between differential expression levels of the target genes in the $\mathrm{T}_{2}$ generation

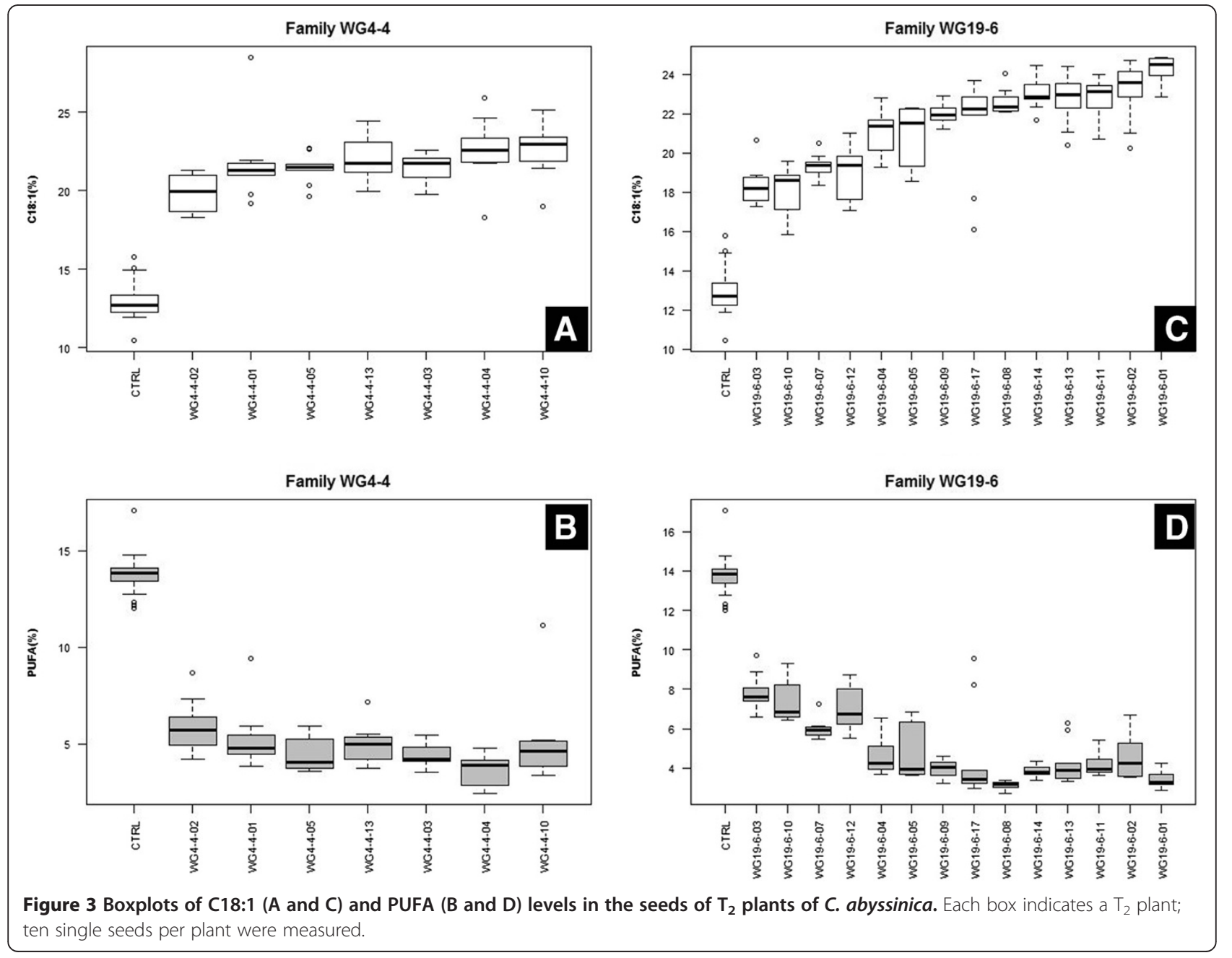




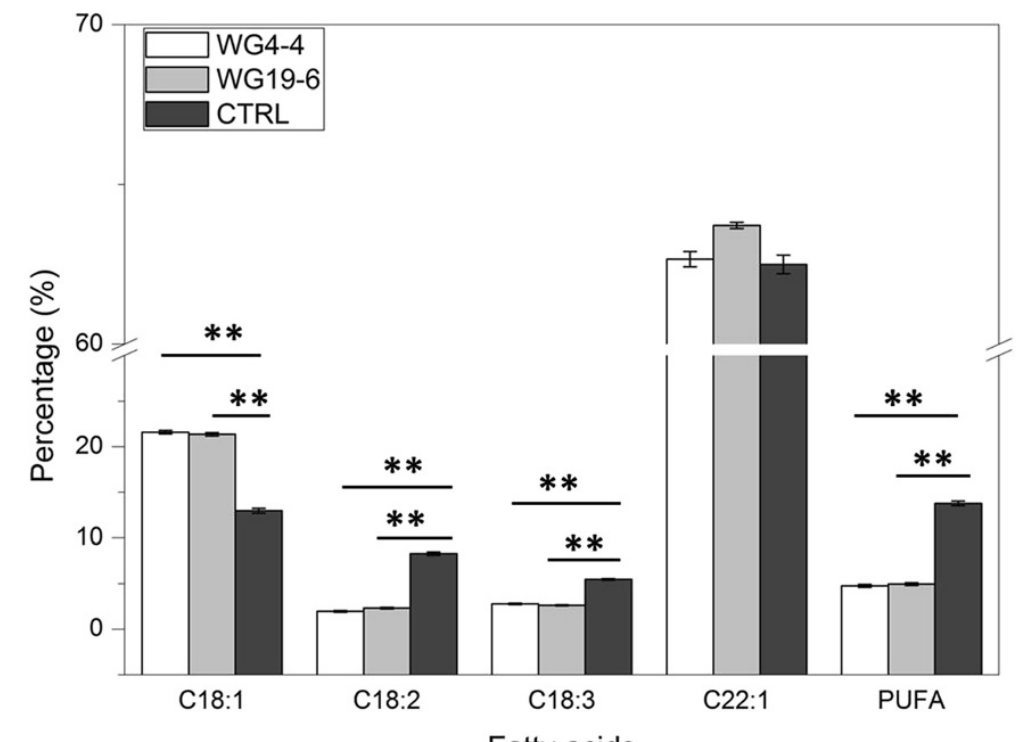

Fatty acids

Figure 4 Comparison of the oil composition in the seeds of $T_{2}$ family WG4-4, WG19-6 and control plants of $C$. abyssinica. The data represent the average of the two families and the control. For each plant, ten single seeds were measured. The bars indicate the comparisons with significant difference. ${ }^{* *}, P<0.01$. Error bar $=$ standard error of mean.

and the oil composition, correlation analysis was carried out. All the correlations and coefficients are plotted in Figure 5. Among the individual $\mathrm{T}_{2}$ plants of WG4-4 and WG19-6 that showed differential CaFAD2 expression levels, some significant correlations were observed for the expression levels of CaFAD2-C1 to -C3, CaFAD3 and CaFAE1. Overall, the expression of CaFAE1 is co-ordinately regulated with both, CaFAD3 $(\mathrm{r}=0.92$, $P<0.001)$ and CaFAD2-C2 $(\mathrm{r}=0.44, P<0.05)$ (Figure 5). These correlations are much stronger in the WG4-4 family $(r=0.943$ and 0.957 respectively). Regarding the oil composition, the contents of both $\mathrm{C} 18: 2$ and $\mathrm{C} 18: 3$

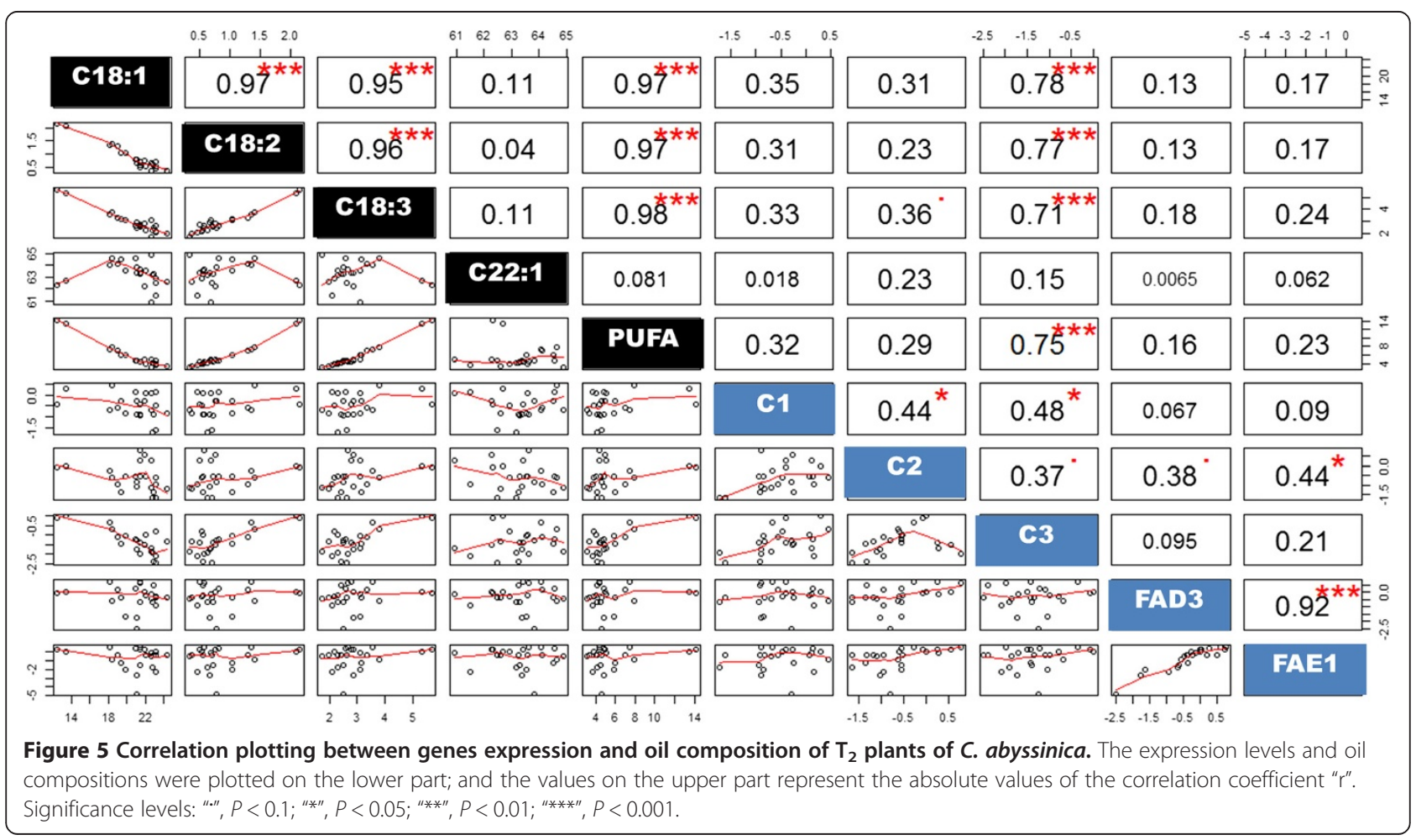


were negatively related $(\mathrm{r}=-0.97$ and -0.95 respectively, $P<0.001)$ to the C18:1 content. However, the C22:1 content was not significantly related to the observed changes in other oil compounds $(P>0.1)$ (Figure 5). In accordance with its function in the oil biosynthesis pathway, the expression level of CaFAD2-C3 is negatively related to the $\mathrm{C} 18: 1$ content $(\mathrm{r}=-0.78, P<0.001)$ and positively related to the contents of $\mathrm{C} 18: 2(\mathrm{r}=0.77, P<0.01)$ and C18:3 ( $r=0.71, P<0.01$ ) (Figure 5). However, there were no correlations found between these fatty acids and the expression of other genes (Figure 5).

\section{Discussion}

C. abyssinica, an under-utilized crop, has limited genetic variation and the improvement of crambe oil by traditional breeding has reached a bottleneck. Recently, using a genetic engineering approach, crambe lines with a desirable oil type have been obtained by silencing CaFAD2 genes [29]. In C. abyssinica, three functional FAD2 genes are present (CaFAD2-C1 to -C3) [32]. Even though the functional defect of one of the family members may be complemented by the other members of the gene family [41], one or few of these family members usually play a predominant role, so that a primary functional analysis of gene family members is normally required prior to mutation breeding [42]. Our study was initiated to characterise the effect of the three functional CaFAD2 genes (CaFAD2-C1 to -C3) to determine the appropriate targets for a targeted mutation approach (TILLING) to develop non-GM crambe lines with novel oil types.

Here we used a construct containing a part of the CaFAD2-C2 sequence, which is $96 \%$ to $97 \%$ identical to the sequences of other expressed CaFAD2 genes, to obtain seed-specific silencing (Napin promoter) of the endogenous $C a F A D 2$ gene family members in crambe. The results proved that one RNAi trigger is able to affect the expression of multiple members of the gene family but in different patterns (Figure 1). In $\mathrm{T}_{2}$-plants, the main FAD2 gene expressed in crambe seeds, CaFAD2-C3, is strongly silenced whereas the two lower expressed genes, CaFAD2-C2 and -C1 (respectively 4 and 100 times lower than CaFAD2-C3 at 20 DAP [32]), are silenced to different levels ranging from strong- to zero silencing. The reason why no reduction of CaFAD2 gene expression was detected in two $\mathrm{T}_{1}$-plants (WG19-5 and WG19-6), which nevertheless showed significant difference for seed-oil composition, is probably due to the heterozygous nature of the seed samples. This can cause that segregating wild-type seeds can be present in the seed bulks used for expression analysis, whereas the oil composition was measured on single seeds. Another possibility is that silencing in these lines took place in the developing seeds at other time points beyond the expression peak at 20 days after pollination (DAP). Indeed, the results of Southern analysis do suggest that the $T_{1}$ plant WG19-6 is heterozygous, because a varying number of transgene insertions was detected in its offspring $\left(\mathrm{T}_{2}\right)$, and also that WG4-4 ( $\mathrm{T}_{1}$-plant) is most likely homozygous because a single transgene insertion was observed in all randomly tested progeny $\left(\mathrm{T}_{2}\right)$ of WG4-4 (Additional file 2).

Over two generations $\left(\mathrm{T}_{1}\right.$ and $\left.\mathrm{T}_{2}\right)$, the expression patterns of CaFAD2 in WG4-4, carrying a single transgene insertion, were similar whereas differences in silencing levels over generations were observed for WG19-6. The multiple transgene insertions in WG19-6 may cause different types of variation in the $T_{2}$ population, which may influence the level of silencing. It is known that trace amount of dsRNA is sufficient to trigger gene silencing [43] and the degree of silencing has no association with insert copy number [44]. Therefore, a plausible explanation to different silencing levels over $T_{1}$ and $T_{2}$ generations of WG19-6 is that a higher level of homozygosity in the seeds of the $T_{2}$ generation leads to a lower number of segregating wild type seeds, which is of importance if the seeds are analysed for expression "in bulk".

The $\mathrm{T}_{2}$ progenies of two FAD2-RNAi plants (WG4-4 and WG19-6) were tested in detail for the level of gene expression of the different CaFAD2 gene family members (Figure $2 \mathrm{~A}$ and $\mathrm{B}$ ) and for oil composition (Figure 3 and 4). The results showed that the silencing of gene expression is stably inherited to the subsequent generation. The stability of RNAi-silencing was also reflected at the seed-oil composition, for instance, in the $T_{2}$-progeny of WG4-4, the C18:1 level was significantly higher and PUFA level was significantly lower than those of the control plants (Table 1 and Figure 3). The values of important individual oil compounds are close to the average of the parental $\mathrm{T}_{1}$-plants for both lines, WG4-4 and WG19-6, indicating that the high $\mathrm{C} 18: 1$ and low PUFA traits were stably transmitted to the subsequent generation.

\section{Correlation and expression regulation analysis}

Compared to CaFAD2-C1 and CaFAD2-C2, CaFAD2-C3 was strong and stably silenced by the seed specific RNAi trigger. On average, silencing of CaFAD2-C3 is constantly significant in both $\mathrm{T}_{2}$ families (offspring of WG4-4 and WG19-6). Although some $\mathrm{T}_{2}$-plants show a clear silencing of CaFAD2-C1 and CaFAD2-C2, the overall family average of these genes are not significantly different from the control for both families. Consequently, the only significant difference in gene expression observed between the two $\mathrm{T}_{2}$ families is the difference in CaFAD2-C2 expression (Figure 2B).

Taken together with the fact that a change in oil composition was found in all $\mathrm{T}_{2}$-plants and that out of the three CaFAD2 genes CaFAD2-C3 is the highest expressor in developing crambe seeds [32], it is most likely that this 
gene plays a direct role in seed oil synthesis. This idea is supported by the study on the seed-oil of individual $\mathrm{T}_{2^{-}}$ plants (Figure 5). Based on the differential silencing of CaFAD2-C2 among $\mathrm{T}_{2}$ plants of both families and the silencing of CaFAD2-C1 observed in several individual $\mathrm{T}_{2}$ plants, there is no clear evidence showing that the expression of these two genes is correlated to changes in the seed oil composition (Figure 5). Furthermore, the changes in oil composition due to CaFAD2-C3-silencing were not complemented in individual $\mathrm{T}_{2}$-plants in which CaFAD2$C 1$ and CaFAD2-C2 were unaffected and expressed to wild-type levels.

The trait of high C18:1 is controlled by quantitative trait loci and thus needs coordinated regulation of multiple loci [21]. Previous studies proved that the FAD2 gene plays an essential role for this trait $[9,38]$. In addition, $F A D 3, F A E 1$ and other loci with minor effect are likely required to establish high C18:1 pool during oil biosynthesis $[21,45]$. In this study, we also investigated the regulation of FAD3 and FAE1 while silencing the CaFAD2 genes. It is known that FAD3 and $F A E 1$ are regulated by abscisic acid [46]. Herein, we found that the expression of $F A E 1$ is positively related to that of $F A D 3$ and one of the FAD2 genes, $C a F A D 2-C 2$ (Figure 5). This finding is consistent to previous studies in which these functionally related genes (FAD3 and FAE1) showed coordinated regulation in Arabidopsis and Brassica napus [46,47]. However, it is unknown why the other two CaFAD2 genes do not show a similar co-regulation with FAD3 and FAE1.

\section{The CaFAD2-C3 gene is a target gene for crambe oil improvement by mutation breeding}

Here, we show that CaFAD2-C3 is the main CaFAD2gene involved in determining the C18:1 and PUFA contents in crambe oil (Figure 5). Embryo tissue is the main compartment of oil storage in crambe seed, so the oil composition in the seeds is mainly determined by that in the embryo. Therefore, CaFAD2-C3 is most likely responsible for fatty acid synthesis in the embryo. The other two genes, CaFAD2-C1 and CaFAD2-C2, might be responsible to change fatty acid contents in other seed compartments (e.g. seed coat and endosperm). For instance in olive (Olea europaea), two FAD2 genes (OeFAD2-1 and OeFAD2-2) are expressed both in two seed compartments, seed coat and embryo. Of these two OeFAD2 genes, the expression of OeFAD2-2 was positively correlated to C18:2 content in the seed coat rather than in the embryo under cold condition [48].

\section{Conclusions}

The finding that the prominent role of CaFAD2-C3 is further substantiated by the observation that the effect on oil-composition caused by CaFAD2-C3 silencing is not restored in individual $\mathrm{T}_{2}$-plants with wild-type expression of CaFAD2-C2 and -C1 respectively. This finding suggests that $C a F A D 2-C 3$ may provide an important target gene for TILLING and mutation breeding aiming at pronounced changes of C18:1 and PUFA in crambe oil.

\section{Methods \\ Plant materials}

C. abyssinica cv. 'Galactica' was previously transformed with an RNAi construct (pWatergate) [29]. This construct contains an inverted repeat (IR) of the CaFAD2C2 coding sequence (355 base pairs for each IR part, 97\% identical to CaFAD2-C1 and $96 \%$ identical to CaFAD2-C3) following a seed specific promoter (Napin) (Additional file 4). The plant used as control was transformed with an empty construct (pRCNG) which contains no genes involved in fatty acids biosynthesis [49]. Two $T_{0}$ lines were used to develop six $T_{1}$ plants/ lines (WG4-3, WG4-4, WG4-5, WG19-4, WG19-5 and WG19-6), grown in the greenhouse with temperature of $22^{\circ} \mathrm{C}$ and photoperiod of $16 \mathrm{~h}$. From these $\mathrm{T}_{1}$ plants, the developing seeds at 20 DAP (days after pollination) were collected for RNA isolation for gene expression analysis and ripe seeds were harvested for oil composition analysis. Seeds of two $\mathrm{T}_{1}$-lines, WG4-4 and WG19-6, were used to produce $T_{2}$-plants. The young leaves of $T_{2}$ plants were collected for Southern analysis. The developing seeds (20 DAP) and ripe seeds of the $\mathrm{T}_{2}$-plants were harvested for RNA isolation and oil composition analysis respectively.

\section{Quantitative reverse transcription PCR (qRT-PCR) and data analysis}

To examine the level of gene-silencing, the expression of the individual crambe FAD2 genes, CaFAD2-C1, CaFAD2$C 2$ and CaFAD2-C3, was measured in six $\mathrm{T}_{1}$ plants (WG4-3, WG4-4, WG4-5, WG19-4, WG19-5 and WG196) on bulks of five to ten developing seeds (20 DAP, days after pollination) and compared to their expression level in the control (plant transformed with an empty construct). The primers specific for the different crambe $F A D 2$ genes were developed based on their sequences in $C$. abyssinica cv. 'Galactica' (GenBank: JX964743, JX964744, JX964745), and the primers of FAD3 and FAE1 were developed on the sequences of AtFAD3 (GenBank: 42570333) and crambe FAE1 (GenBank: 60543786). The gene $\beta$-actin 2 (GenBank: 20465834) was used as a reference gene. All primers used in qRT-PCR are listed in Additional file 5. Total RNA was extracted from bulked seeds of $T_{1}$-and $\mathrm{T}_{2}$-plants respectively (5 to 10 seeds per $\mathrm{T}_{1}$-plant and per $\mathrm{T}_{2}$-plant, $20 \mathrm{DAP}$ ) with RNeasy Plant Mini Kits (Qiagen, Germany) according to the manufacturer's instructions. The isolated RNA was treated with RNase-free TURBO DNase (Ambion, USA) to remove residual genomic DNA. First-strand cDNA was synthesized in $20 \mu \mathrm{l}$ from $1 \mu \mathrm{g}$ of 
total RNA with iScript ${ }^{\text {tm }}$ cDNA Synthesis Kit (Bio-rad, USA), in parallel $1 \mu \mathrm{g}$ of RNA of each sample was treated in the same way but without adding reverse transcriptase, as negative controls (RT-). The cDNA was $20 \times$ diluted and used as templates for real-time PCR. The PCR reaction contains $2 \mu$ templates, $5 \mu$ SYBR Green Super Mix (Bio-rad, USA), and $1 \mu \mathrm{l}$ of each of the forward and reverse primers $(3 \mu \mathrm{M})$ in total $10 \mu \mathrm{l}$ reaction. Cycling conditions were 1 cycle at $95^{\circ} \mathrm{C}$ for $3 \mathrm{~min}$ followed by 30 cycles at $95^{\circ} \mathrm{C}$ for $10 \mathrm{~s}, 60^{\circ} \mathrm{C}$ for $1 \mathrm{~min}$, then a final melt step from $65^{\circ} \mathrm{C}$ to $95^{\circ} \mathrm{C}$ ramp with $0.5^{\circ} \mathrm{C}$ increments per cycle to monitor specificity. PCR reactions were performed in triplicate. The expression of each replicate was normalized by the reference gene, $\beta$-actin 2 , which has shown to be stably expressed in both, crambe seedlings under arsenate stress and various Brassica napus cultivars $[47,50]$. The relative expression level of each replicate was calculated according to the comparative CT method (User bulletin no. 2, ABI PRISM 7700 Sequence Detection System, December 1997; Perkin-Elmer, Applied Biosystems). The mean of three replicates represents the relative expression level of a line.

\section{Oil composition and correlation analysis}

The fatty acid (FA) fraction was extracted from single crambe seeds, and fatty acid methyl esters (FAMEs) were analysed using gas chromatograph (GC, column/DB-23, Agilent). For each $\mathrm{T}_{1}$-plant 20 , and for each $\mathrm{T}_{2}$-plant 10 individual ripe seeds were collected respectively per plant for fatty acid extraction. The pods of single seeds were removed and then crushed in a plastic tube with screw cap and $300 \mu \mathrm{l}$ of hexane and $40 \mu \mathrm{l}$ of $\mathrm{KOH}(5 \mathrm{M}) /$ methanol were added, followed by vortexing and heating samples at $60^{\circ} \mathrm{C}$ for $6 \mathrm{~min}$. The samples were then allowed to cool down to room temperature before centrifugation at $3000 \mathrm{rpm}$ for $5 \mathrm{~min}$, the upper layer was used for GC. The extraction $(1 \mu \mathrm{l})$ was injected into GC with split ratio $1: 20$ and the condition of oven was $180^{\circ} \mathrm{C}$ for $10 \mathrm{~min}$, ramp to $240^{\circ} \mathrm{C}$ for $7 \mathrm{~min}$ with $7.5^{\circ} \mathrm{C}$ increments per min. The mean percentages of FA compounds of each line were calculated from the average of 20 or 10 single seed values. The data of gene expression and oil composition in the seeds of $\mathrm{T}_{2}$ generation were used for correlation analysis. These data were plotted and a Pearson correlation was run to determine correlation with $\mathrm{R}$ package [51].

\section{Southern analysis}

Genomic DNA was isolated from young leaves of $T_{2}$ plants with the method described by Aldrich and Cullis [52] but with $1 \%(\mathrm{w} / \mathrm{v})$ polyvinylpyrrolidone-10 in the DNA extraction buffer. A probe (686 base pairs) was designed on the nptII gene in the WG construct and labelled with $\left[{ }^{32} \mathrm{P}\right] \mathrm{ATP}$. The primers for nptII probe preparation are listed in Additional file 5.
For copy number determination, a total of $40 \mu \mathrm{g}$ of DNA extracted was digested overnight with Dra I, an enzyme that cuts the T-DNA in a site outside the probe sequence, fractionated on $0.8 \%$ agarose gel and transferred to Hybond $\mathrm{N}+$ membrane (Amersham Biosciences, UK) according to the manufacturer's recommendations. The procedures of hybridization and visualization were performed as described by Cheng et al. [32] .

\section{Additional files}

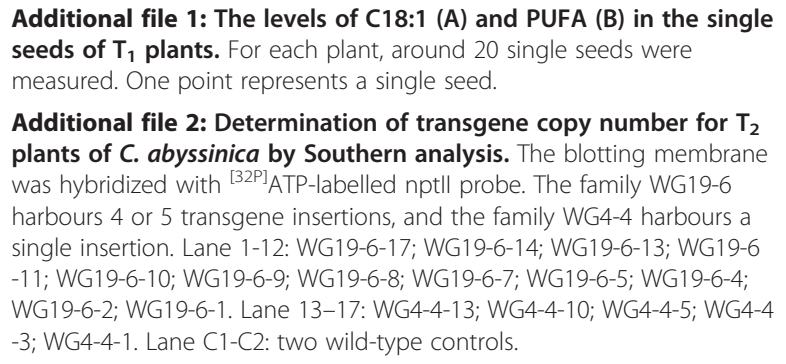
plants of C. abyssinica by Southern analysis. The blotting membrane was hybridized with ${ }^{[32 \mathrm{P}]}$ ATP-labelled nptll probe. The family WG19-6 harbours 4 or 5 transgene insertions, and the family WG4-4 harbours a single insertion. Lane 1-12: WG19-6-17; WG19-6-14; WG19-6-13; WG19-6 -11; WG19-6-10; WG19-6-9; WG19-6-8; WG19-6-7; WG19-6-5; WG19-6-4; WG19-6-2; WG19-6-1. Lane 13-17: WG4-4-13; WG4-4-10; WG4-4-5; WG4-4 -3; WG4-4-1. Lane C1-C2: two wild-type controls.

Additional file 3: The plot of oil composition (C18:1 vs. PUFA) in the seeds of $T_{2}$ plants of family WG4-4, WG19-6 and the control (CTRL). Each point represents a single seed of $T_{2}$-plant.

Additional file 4: Schematic diagram of T-DNA region of the RNAi construct (not to scale). LB and RB, T-DNA left border and right border, respectively. Napin Promoter, seed specific promoter from Brassica napus. The attB1 and attB2, recombination sites used in BP reaction of Gateway". CaFAD2-IF and CaFAD2-IR, 355 base pairs inverted repeats of crambe FAD2-C2 sequence in forward and reverse orientations, the sequence identities to the genes CaFAD2-C1,-C2 and-C3 are 96\%, 99\% and 96\% respectively. nptIl, neomycin hosphotransferase II gene. The broken line represents the sequence that forms stem in hairpin RNA. The arrow indicated npt II probe (686 base pairs) for hybridization in this study.

Additional file 5: The primers used in this study.

\section{Competing interests}

The authors declare that they have no competing interests.

\section{Authors' contributions}

JC, EMJS and ENVL designed the experiments. JC carried out the expression analysis and wrote the draft manuscript; EMJS performed Southern analysis and amended the manuscript. LHZ and JG provided transgenic plants and commented the manuscript. ACD and WQ performed GC analysis. BH, FAK and RGFV convinced the study and participated in amending the draft manuscript. All authors read and approved the final manuscript.

\section{Acknowledgements}

This work was supported by the EU-project ICON (Industrial crops producing added value oils for novel chemicals) with co-funding of a Dutch R\&D program Biobased Economy (Kennis Basis Biobased Economy).

\section{Author details}

${ }^{1}$ Wageningen UR Plant Breeding, P.O. Box 16, 6700, AA Wageningen, The Netherlands. ${ }^{2}$ College of Life Science, Hubei University, Wuhan, People's Republic of China. ${ }^{3}$ Plant Breeding and Biotechnology, Swedish University of Agricultural Science, Alnarp, Sweden. ${ }^{4}$ Institute for Biology I-Botany, RWTH Aachen University, Aachen, Germany.

Received: 8 May 2013 Accepted: 24 September 2013 Published: 1 October 2013 
References

1. Mastebroek H, Wallenburg S, Van Soest L: Variation for agronomic characteristics in crambe (Crambe abyssinica Hochst. ex Fries). Ind Crops Prod 1994, 2(2):129-136.

2. Mietkiewska E, Brost JM, Giblin EM, Barton DL, Taylor DC: Cloning and functional characterization of the fatty acid elongase 1 (FAE1) gene from high erucic Crambe abyssinica cv: prophet. Plant Biotechnol J 2007, 5(5):636-645.

3. Falasca S, Flores N, Lamas M, Carballo SM, Anschau A: Crambe abyssinica: an almost unknown crop with a promissory future to produce biodiesel in Argentina. Int J Hydrog Energy 2010, 35(11):5808-5812.

4. Cuperus FP, Boswinkel G, Derksen JTP: The processing of new oilseed crops-an economic evaluation. J Am Oil Chem Soc 1996, 73(12):1635-1640.

5. Carlsson A, Clayton D, Salentijn E, Toonen M, Stymne S, Dyer W, Bowles D: Oil crop platforms for industrial uses. In Outputs from the EPOBIO projects. Newbury: York: CPL Press; 2007:146.

6. Youping W, Peng L: Intergeneric hybridization between Brassica species and Crambe abyssinica. Euphytica 1998, 101(1):1-7.

7. Zanetti F, Vamerali T, Bona S, Mosca G: Can we "cultivate" erucic acid in southern Europe? Ital J Agron 2006, 1(1):3-10.

8. Jadhav A, Katavic V, Marillia EF, Michael Giblin E, Barton DL, Kumar A Sonntag C, Babic V, Keller WA, Taylor DC: Increased levels of erucic acid in Brassica carinata by co-suppression and antisense repression of the endogenous FAD2 gene. Metab Eng 2005, 7(3):215-220.

9. Yang Q, Fan C, Guo Z, Qin J, Wu J, Li Q, Fu T, Zhou Y: Identification of FAD2 and FAD3 genes in Brassica napus genome and development of allele-specific markers for high oleic and low linolenic acid contents. TAG Theor App/ Genet 2012, 125(4):715-729.

10. Ma F, Hanna MA: Biodiesel production: a review. Bioresour Technol 1999, 70(1):1-15.

11. Pham AT, Shannon JG, Bilyeu KD: Combinations of mutant FAD2 and FAD3 genes to produce high oleic acid and low linolenic acid soybean oil. TAG Theor Appl Genet 2012, 125(3):503-515

12. Ohlrogge JB: Design of new plant products: engineering of fatty acid metabolism. Plant Physiol 1994, 104(3):821-826.

13. Ohlrogge J: Lipid biosynthesis. Plant Cell 1995, 7(7):957-970.

14. Barret P, Delourme R, Renard M, Domergue F, Lessire R, Delseny M, Roscoe T: A rapeseed FAE1 gene is linked to the E1 locus associated with variation in the content of erucic acid. TAG Theor App/ Genet 1998, 96(2):177-186.

15. Rossak M, Smith M, Kunst L: Expression of the FAE1 gene and FAE1 promoter activity in developing seeds of Arabidopsis thaliana. Plant Mol Biol 2001, 46(6):717-725.

16. Blacklock BJ, Jaworski JG: Studies into factors contributing to substrate specificity of membrane-bound 3-ketoacyl-CoA synthases. Eur J Biochem 2002, 269(19):4789-4798

17. Salas JJ, Martínez-Force E, Garcés R: Very long chain fatty acid synthesis in sunflower kernels. J Agric Food Chem 2005, 53(7):2710-2716.

18. Miquel M: Arabidopsis mutants deficient in polyunsaturated fatty acid synthesis: biochemical and genetic characterization of a plant oleoylphosphatidylcholine desaturase. J Biol Chem 1992, 267(3):1502-1509.

19. Löhden I, Frentzen M: Role of plastidial acyl-acyl carrier protein: glycerol 3-phosphate acyltransferase and acyl-acyl carrier protein hydrolase in channelling the acyl flux through the prokaryotic and eukaryotic pathway. Planta 1988, 176(4):506-512.

20. Andre C, Haslam RP, Shanklin J: Feedback regulation of plastidic acetylCoA carboxylase by 18: 1-acyl carrier protein in Brassica napus. Proc Natl Acad Sci 2012, 109(25):10107-10112

21. Guan M, Li X, Guan C: Microarray analysis of differentially expressed genes between Brassica napus strains with high-and low-oleic acid contents. Plant Cell Rep 2012, 31(5):929-943.

22. Liu Q, Singh SP, Green AG: High-stearic and high-oleic cottonseed oils produced by hairpin RNA-mediated post-transcriptional gene silencing. Plant Physiol 2002, 129(4):1732-1743.

23. Sivaraman I, Arumugam N, Sodhi YS, Gupta V, Mukhopadhyay A, Pradhan AK, Burma PK, Pental D: Development of high oleic and low linoleic acid transgenics in a zero erucic acid Brassica juncea L.(Indian mustard) line by antisense suppression of the fad2 gene. Mol Breed 2004, 13(4):365-375.

24. Wesley SV, Helliwell CA, Smith NA, Wang MB, Rouse DT, Liu Q, Gooding PS, Singh SP, Abbott D, Stoutjesdijk PA: Construct design for efficient, effective and high-throughput gene silencing in plants. Plant J 2001, 27(6):581-590.
25. Rathore KS, Sundaram S, Sunilkumar G, Campbell LM, Puckhaber L, Marcel S, Palle SR, Stipanovic RD, Wedegaertner TC: Ultra-low gossypol cottonseed: generational stability of the seed-specific, RNAi-mediated phenotype and resumption of terpenoid profile following seed germination. Plant Biotechnol J 2012, 10(2):174-183

26. Peng Q, Hu Y, Wei R, Zhang Y, Guan C, Ruan Y, Liu C: Simultaneous silencing of FAD2 and FAE1 genes affects both oleic acid and erucic acid contents in Brassica napus seeds. Plant Cell Rep 2010, 29(4):317-325

27. Li X, Ahlman A, Yan X, Lindgren H, Zhu LH: Genetic transformation of the oilseed crop Crambe abyssinica. Plant Cell, Tissue Organ Cult 2010 100(2):149-156.

28. Chhikara S, Dutta I, Paulose B, Jaiwal PK, Dhankher OP: Development of an Agrobacterium-mediated stable transformation method for industrial oilseed crop Crambe abyssinica 'BelAnn'. Ind Crops Prod 2012 37(1):457-465

29. Li X, Van Loo EN, Gruber J, Fan J, Guan R, Frentzen M, Stymne S, Zhu LH: Development of ultra-high erucic acid oil in the industrial oil crop Crambe abyssinica. Plant Biotechnol J 2012, 10(7):862-870.

30. Gómez-Galera S, Twyman RM, Sparrow PAC, Van Droogenbroeck B, Custers $R$, Capell T, Christou P: Field trials and tribulations-making sense of the regulations for experimental field trials of transgenic crops in Europe. Plant Biotechnol J 2012, 10(5):511-523.

31. Lessman K, Meier $\mathrm{V}$ : Agronomic evaluation of crambe as a source of oil. Crop Sci 1972, 12(2):224-227

32. Cheng J, Salentijn E, Huang B, Krens F, Dechesne A, Visser R, Van Loo E: Isolation and characterization of the omega- 6 fatty acid desaturase (FAD2) gene family in the allohexaploid oil seed crop Crambe abyssinica Hochst. Mol Breed. 2013, 32(3):517-531.

33. McCallum CM, Comai L, Greene EA, Henikoff S: Targeted screening for induced mutations. Nat Biotechnol 2000, 18(4):455-457.

34. Chen L, Huang L, Min D, Phillips A, Wang S, Madgwick PJ, Parry MAJ, Hu YG: Development and characterization of a new TILLING population of common bread wheat (Triticum aestivum L.). PLOS ONE 2012, 7(7):e41570.

35. Slade AJ, McGuire C, Loeffler D, Mullenberg J, Skinner W, Fazio G, Holm A, Brandt KM, Steine MN, Goodstal JF, et al: Development of high amylose wheat through TILLING. BMC plant biology 2012, 12(1):69.

36. Auld DL, Heikkinen MK, Erickson DA, Sernyk JL, Romero JE: Rapeseed mutants with reduced levels of polyunsaturated fatty acids and increased levels of oleic acid. Crop science 1992, 32(3):657-662.

37. Pham AT, Lee JD, Shannon JG, Bilyeu K: Mutant alleles of FAD2-1A and FAD2-1B combine to produce soybeans with the high oleic acid seed oil trait. BMC plant biology 2010, 10(1):195.

38. Pham AT, Lee JD, Shannon JG, Bilyeu KD: A novel FAD2-1 A allele in a soybean plant introduction offers an alternate means to produce soybean seed oil with $85 \%$ oleic acid content. TAG Theor App/ Genet 2011, 123(5):793-802.

39. Tanhuanpää P, Vilkki J, Vihinen M: Mapping and cloning of FAD2 gene to develop allele-specific PCR for oleic acid in spring turnip rape (Brassica rapa ssp. oleifera). Mol Breed 1998, 4(6):543-550.

40. Beló A, Zheng P, Luck S, Shen B, Meyer DJ, Li B, Tingey S, Rafalski A: Whole genome scan detects an allelic variant of fad 2 associated with increased oleic acid levels in maize. Mol Genet Genomics 2008, 279(1):1-10.

41. Lynch $\mathrm{M}$, Conery JS: The evolutionary fate and consequences of duplicate genes. Science 2000, 290(5494):1151-1155.

42. Wang $N$, Wang $Y$, Tian F, King GJ, Zhang C, Long Y, Shi L, Meng J: A functional genomics resource for Brassica napus: development of an EMS mutagenized population and discovery of FAE1 point mutations by TILLING. New Phytol 2008, 180(4):751-765

43. Fire A: RNA-triggered gene silencing. Trends Genet 1999, 15(9):358-363

44. Li J, Brunner AM, Shevchenko O, Meilan R, Ma C, Skinner JS, Strauss SH: Efficient and stable transgene suppression via RNAi in field-grown poplars. Transgenic research 2008, 17(4):679-694

45. Jagannath A, Sodhi YS, Gupta V, Mukhopadhyay A, Arumugam N, Singh I, Rohatgi S, Burma PK, Pradhan AK, Pental D: Eliminating expression of erucic acid-encoding loci allows the identification of "hidden" QTL contributing to oil quality fractions and oil content in Brassica juncea (Indian mustard). Theor App/ Genet 2011, 122(6):1091-1103.

46. Ruuska SA, Girke T, Benning C, Ohlrogge JB: Contrapuntal networks of gene expression during Arabidopsis seed filling. Plant Cell 2002, 14(6):1191-1206. 
47. Hu Y, Wu G, Cao Y, Wu Y, Xiao L, Li X, Lu C: Breeding response of transcript profiling in developing seeds of Brassica napus. BMC Mol Biol 2009, 10(1):49.

48. D'Angeli S, Falasca G, Matteucci M, Altamura MM: Cold perception and gene expression differ in Olea europaea seed coat and embryo during drupe cold acclimation. New Phytol 2013, 197(1):123-138.

49. Schaart JG, Krens FA, Pelgrom KTB, Mendes O, Rouwendal GJA: Effective production of marker-free transgenic strawberry plants using inducible site-specific recombination and a bifunctional selectable marker gene. Plant Biotechnol J 2004, 2(3):233-240.

50. Paulose B, Kandasamy S, Dhankher OP: Expression profiling of Crambe abyssinica under arsenate stress identifies genes and gene networks involved in arsenic metabolism and detoxification. BMC plant biology 2010, 10(1):108.

51. R Core Team: $R$ : A language and environment for statistical computing. Vienna, Austria: R Foundation for Statistical Computing; 2012. ISBN 3-900051-07-0, URL http://www.R-project.org/.

52. Aldrich J, Cullis CA: RAPD analysis in flax: optimization of yield and reproducibility using klen Taq 1 DNA polymerase, chelex 100, and gel purification of genomic DNA. Plant Mol Biol Report 1993, 11(2):128-141.

doi:10.1186/1471-2229-13-146

Cite this article as: Cheng et al:: Functional analysis of the omega- 6 fatty acid desaturase (CaFAD2) gene family of the oil seed crop Crambe abyssinica. BMC Plant Biology 2013 13:146.

\section{Submit your next manuscript to BioMed Central and take full advantage of:}

- Convenient online submission

- Thorough peer review

- No space constraints or color figure charges

- Immediate publication on acceptance

- Inclusion in PubMed, CAS, Scopus and Google Scholar

- Research which is freely available for redistribution 